\title{
GEOMETRIC HARDY INEQUALITIES VIA INTEGRATION ON FLOWS
}

\section{PASCHALIS}

Abstract. We introduce a geometric approach of integration along integral curves for functional inequalities involving directional derivatives in the general context of differentiable manifolds that are equipped with a volume form. We focus on Hardy-type inequalities and the explicit optimal Hardy potentials that are induced by this method. We then apply the method to retrieve some known inequalities and establish some new ones.

Mathematics subject classification (2020): Primary 35J75, 58J05; Secondary 35R01, 35J62.

Keywords and phrases: Hardy inequality, directional derivative, vector field, flow, manifold.

\section{REFERENCES}

[1] F. G. Avkhadiev, R. V. MaKarov, Hardy Type Inequalities on Domains with Convex Complement and Uncertainty Principle of Heisenberg, Lobachevskii J. Math 40, 1250-1259 (2019).

[2] Alexander A. Balinsky, W. Desmond Evans, Roger T. Lewis, The Analysis and Geometry of Hardy's Inequality, (2015), Springer International Publishing.

[3] Gerassimos Barbatis \& Stathis Filippas \& Achilles Tertikas, Tertikas A unified approach to improved Lp Hardy inequalities with best constants, Transactions of the American Mathematical Society (2003).

[4] Elvise Berchio, Lorenzo D’ Ambrosio, Debdip Ganguly, Gabriele Grillo, Improved Lp-Poincaré inequalities on the hyperbolic space, Nonlinear Analysis, vol. 157, 2017, pages 146-166.

[5] F. CHIACCHIO AND T. RICCIARDI, Some sharp Hardy inequalities on spherically symmetric domains, Pac. J. Math. 242, no. 1 (2009), 173-187.

[6] Lorenzo D' Ambrosio, Serena Dipierro, Hardy inequalities on Riemannian manifolds and applications, Annales de l'Institut Henri Poincare (C) Non Linear Analysis, vol. 31, issue 3, 2014, pages 449-475.

[7] D. Goel, Y. Pinchover, AND G. Psaradkis, On weighted Lp-Hardy inequality on domains in Rn, to appear in a special issue dedicated to Shmuel Agmon, Pure Appl. Funct. Anal., arXiv: 2012.12860.

[8] Kombe, ISMAIL, AND MurAd ÖZAYdin, Improved Hardy and Rellich inequalities on Riemannian manifolds, transactions of the American Mathematical Society, vol. 361, no. 12, 2009, pp. 6191-6203, JSTOR, www. jstor.org/stable/40590795, Accessed 19 Apr. 2021.

[9] ALEXANDRU KRISTÁLY, Sharp uncertainty principles on Riemannian manifolds: the influence of curvature, Journal de Mathématiques Pures et Appliquées, vol. 119, 2018, pages 326-346.

[10] John M. LeE, Introduction to Smooth Manifolds, Second Edition, Springer Science+Business Media New York, 2003.

[11] M. Marcus, V. J. Mizel And Y. Pinchover, On the best constant for Hardy's inequality in $\mathbb{R}^{n}$, Trans. Amer. Math. Soc. 350 (1998), 3237-3255.

[12] X. Sun, F. PAN,, Hardy type inequalities on the sphere, J. Inequal. Appl. 2017, 148 (2017). 